\title{
Isotretinoína y colitis ulcerosa
}

\section{Isotretinoin and ulcerous colitis}

\author{
E. Borobio ${ }^{1}$, A. Arín' ${ }^{1}$ A. Valcayo ${ }^{2}$, M. Iñarrairaegui ${ }^{1}$, O. Nantes ${ }^{1}$, C. Prieto ${ }^{1}$
}

\section{RESUMEN}

La isotretinoína (Roacután ${ }^{\circledR}$ ), fármaco derivado del ácido retinoico, es utilizado en dermatología como tratamiento para diversos tipos de acné. Su mecanismo de acción a nivel dermo-epidérmico aún no ha sido completamente esclarecido.

Existen en la literatura actual diversos estudios en los que se ha documentado una relación, proporcional o inversa, entre la toma de isotretinoína y el debut o reactivación de una enfermedad inflamatoria intestinal. Aunque se han propuesto diversas hipótesis por las cuales este fármaco podría actuar sobre la mucosa intestinal en la patogénesis de la enfermedad, ninguna de ellas ha sido concluyente.

Presentamos aquí un nuevo caso en el que pudo evidenciarse la mencionada relación, por lo que creemos conveniente recordar que ante un paciente con síntomas sospechosos de enfermedad inflamatoria intestinal en tratamiento activo con este isómero, debe realizarse un procedimiento endoscópico para descartarla.

Palabras clave. Isotretinoína. Colitis ulcerosa. Enfermedad inflamatoria intestinal.

\section{ABSTRACT}

Isotretinoin is a drug obtained from retinoid acid, often used for the treatment of different types of acne. The way it acts on the dermo-epidermis has not been clearly described.

Different studies reporting a relation, proportional or inverse, between isotretinoin and the occurrence or reactivation of an inflammatory bowel disease (IBD) have been found in the literature. Although there are several hypotheses about how this substance can affect the bowel mucous in the pathogenesis of the disease, none of them are definitive.

We present a new case where the mentioned relation was evident. Therefore we suggest that when a patient treated with this isomer presents symptoms suggesting IBD, an endoscopic procedure should be performed in order to rule out inflammatory mucous changes.

Key words. Isotretinoin. Ulcerative colitis. Inflammatory bowel disease.

An. Sist. Sanit. Navar. 2004; 27 (2): 241-243.

1. Servicio de Aparato Digestivo. Hospital de Navarra. Pamplona.

2. Servicio de Dermatología. Hospital de Navarra. Pamplona.

Aceptado para su publicación el 24 de marzo de 2004.

\section{Correspondencia:}

Erika Borobio Aguilar

Servicio de Aparato Digestivo

Hospital de Navarra

C/ Irunlarrea, 3

31008 Pamplona

Tfno: 848422114 


\section{INTRODUCCIÓN}

La isotretinoína (Roacután) es un esteroisómero sintético del ácido retinoico, que está indicado en el tratamiento del acné quístico y acné conglobata. Su mecanismo de acción no ha sido aclarado en su totalidad. Su acción terapéutica está relacionada con la supresión de la actividad de las glándulas sebáceas con reducción del tamaño de las mismas, así como con un efecto antiinflamatorio al nivel de la piel.

En los últimos años se han descrito casos en los cuales, durante un tratamiento con isotretinoína, se ha producido el debut o la recaída de una enfermedad inflamatoria intestinal, sobre todo de una colitis ulcerosa. Esta relación no ha sido suficientemente aclarada por lo que, hoy por hoy, hay muchas incógnitas que están sin resolver.

Describimos un nuevo caso de la posible implicación de este fármaco en el debut de una colitis ulcerosa.

\section{CASO CLÍNICO}

Presentamos el caso de una mujer de 18 años que consultó por rectorragia de 20 días de evolución, aparentemente distal, de escasa cuantía, sin otros síntomas acompañantes. La paciente venía siendo tratada de acné pápulo-pustuloso con Roacután ${ }^{\circledR}$, a la dosis de 10 mg, tres veces al día, desde hacía 4,5 meses. Practicada una rectoscopia, se apreció una ampolla rectal con mucosa eritematosa y granujienta, sin úlceras ni sangrado espontáneo o al roce. Las biopsias fueron informadas de inflamación banal. El cuadro clínico fue etiquetado de rectitis inespecífica y no se instauró tratamiento al remitir la rectorragia de forma espontánea tras la suspensión por parte del Servicio de Dermatología del tratamiento con Roacután ${ }^{\circledR}$. A los tres meses de haber suspendido la isotretinoína presentó diarreas acompañadas de presencia de sangre en las heces. Practicada una colonoscopia, los hallazgos apreciados fueron sugestivos de una colitis ulcerosa de intensidad leve-moderada que se extendía desde recto hasta ángulo esplénico, siendo la histología compatible con colitis ulcerosa. Se instauró tratamiento combinado con mesalazina oral y tópica con buena respuesta.

\section{DISCUSIÓN}

La isotretinoína ha sido invocada como potencial agente desencadenante de brotes de inicio o reagudización de enferme- dad inflamatoria intestinal. En la mayoría de los casos descritos el comienzo de los síntomas intestinales se ha producido durante el tratamiento, si bien hay casos descritos en los que se observa un largo periodo de latencia de meses o años, tras finalizar el tratamiento ${ }^{1}$. Sin embargo, la relación causal de la isotretinoína con la enfermedad inflamatoria intestinal es motivo de controversia ${ }^{5}$. Así, mientras varios autores han reportado casos de enfermedad inflamatoria intestinal en los que la plausibilidad de la relación entre isotretinoína y enfermedad inflamatoria intestinal quedaba suficientemente argumentada ${ }^{1-4}$, otros autores no han observado este efecto adverso del fármaco en amplios grupos de pacientes tratados, incluyendo pacientes con enfermedad inflamatoria intestinal previamente diagnosticada, que no mostraron empeoramiento o reagudización de su enfermedad, a pesar del tratamiento con isotretinoína ${ }^{6,7}$.

En nuestro caso, aplicando el algoritmo de Karsh-Lassagna ${ }^{8}$ para valorar la imputabilidad de un fármaco en una reacción adversa, encontramos una evidente secuencia temporal, la existencia de referencias previas del mismo efecto adverso, así como la mejoría inicial tras la retirada del fármaco, lo que situaría el grado de imputabilidad en la categoría de posible. La reagudización posterior, sin reintroducción del fármaco, cabe atribuirla al comportamiento habitual de la colitis ulcerosa como enfermedad crónica y recurrente.

El mecanismo por el cual la isotretinoína puede inducir enfermedad inflamatoria intestinal es desconocido. Se han sugerido varias hipótesis patogénicas, como inducción de apoptosis, estimulación de linfocitos $\mathrm{T}$ killer, disturbios en el trofismo del epitelio intestinal o disrupción de la síntesis de glicoproteinas ${ }^{1}$, así como un mecanismo de idiosincrasia en posible relación a algún déficit enzimático aún no descrito²

Concluimos que ante la presentación de rectorragias en pacientes tratados con isotretinoína, convendría realizar una endoscopia digestiva baja con el fin de descartar la existencia de una colitis ulcerosa, debiendo usarse este fármaco con 
cautela en pacientes ya diagnosticados de enfermedad inflamatoria intestinal.

\section{BIBLIOGRAFÍA}

1. PROKOP LD. Isotretinoin: posible component cause of inflammatory bowel disesase (letter). Am J Gastroenterol 1999; 94: 2568.

2. Melki M, Pouderoux P, Pignodel C, Balmes JL. Granulomatous colitis likely induced by isotretinoin. Gastroenterol Clin Biol 2001; 25: 433-435.

3. RENIERS DE, HOWARD JM. Isotretinoin-induced inflammatory disease in an adolescent. Ann Pharmacother 2001; 35: 1214-1216.

4. Deplaix P, Barthelemy C, Vedrines P, Perrot JL, Lanthier K, Pignato F et al. Probable acute hemorrhagic colitis caused by isotretinoin with a test of repeated administration. Gastroenterol Clin Biol 1996; 20: 113-114.

5. Gold MH, Roenigk HH JR, Vanagunas A. The retinoids and inflammatory bowel disease. Arch Dermatol 1988; 124: 325-326.

6. GODFREY KM, JAMES MP. Treatment of severe acne with isotretinoin in patients with inflammatory bowel disease. Br J Dermatol 1990; 123: 653-655.

7. SCHLEICHER SM. Oral isotretinoin and inflammatory bowel disease. J Am Acad Dermatol 1985; 15: 834-835.

8. KARSH-LASAGNA. Toward the operational identification of adverse drug reaction. Clin Pharmacl Ther 1997; 21: 247-254. 
